\title{
Agricultural Environment Information Management
}

Xi Cai, Chongqing Technology and Business Institute, Chongqing, China

\begin{abstract}
ZigBee communication technology was used to design the internal information transmission system of intelligent gateway in the monitoring system. Finally, the system was used to detect the changes of temperature and humidity in the environment in real time for verification. The results showed that Amazon AWS cloud service platform was used to build the monitoring system, while the application layer used Spring MVC system framework in J2EE platform to realize the storage of perception data, control of equipment, and analysis of data, and developed the DEMO system by using Amazon DynamoDB NoSQL database and RDS database to jointly store data. The results of this study show that the agricultural environment IOT monitoring system based on cloud computing can better realize the monitoring of relevant parameters in the crop environment, and can lay a foundation for the development of agricultural technology of follow-up facilities.
\end{abstract}

\section{KEYWORDS}

Agricultural Environment, Cloud Computing, Intelligent Gateway, The IoT, ZigBee Communication Technology

\section{INTRODUCTION}

With only $7 \%$ of the world's arable land, China feeds $22 \%$ of the world's population. According to statistics, the annual use of pesticides in China is about 1.8 million tons, far beyond the world average level of pesticide use, which greatly aggravates the pollution of soil and water in China. Lai (2017) and Liu et al. (2018) revealed that in order to realize the sustainable development of agriculture, the development of facility agriculture and modern agriculture has gradually attracted extensive attention. Facility agriculture can improve the air temperature, air humidity, carbon dioxide concentration, soil humidity and other factors directly affecting the growth of crops in the growing environment so that it can maintain a relatively stable and controllable range. According to Wang et al. (2018), this technology ensures the growth needs of the crops, provides the optimum environment, and achieves the high yield of the crops. Modern agricultural technology is a method to apply the advanced science and technology, production materials, and manage the agricultural production process with scientific management means. With the continuous development of computer technology and the extensive application of network information technology, based on the world Internet, the research and application of "IOT" technology in the "third information technology revolution" has reached a climax. At present, the application of IOT technology for agricultural information management has made great achievements. 
IoT technology is a method that can realize intelligent information management and control through network perception, identification, transmission, analysis, and control. In the modern agricultural production process, Zhang et al. (2017) pointed out that IoT technology is used to detect the light intensity and other data in the production environment of crops, which are processed and transmitted to the cloud platform of agricultural IoT. A study showed that cloud computing in the cloud platform can analyze, process and display the obtained agricultural information data in real-time. And intelligent decision feedback was made to the user to achieve real-time monitoring of agricultural production process and automation and intelligent management of production facilities, so that crops have been in a relatively superior and appropriate environment for production, and thereby obtain the maximum production benefits (Wang et al., 2017).

To respond to and promote the development of agricultural IoT in China, the integrated application of IOT technology and cloud computing platform in modern agricultural production process are realized. In this research, the functional structure of agricultural environment IoT monitoring system was designed and constructed, and the hierarchical framework of agricultural environment IoT monitoring system based on cloud computing was designed. Finally, the intelligent gateway system in the agricultural environment IoT monitoring system was designed based on ZigBee technology. The results lay a foundation for the study of real-time detection of the growing environment of crops by using the IoT technology and cloud computing platform in the process of agricultural production.

\section{LITERATURE REVIEW}

The IoT technology can also be applied to the monitoring and utilization of water resources, soil and air, and other agricultural ecological environment monitoring. The concept of smart agriculture came along. Through the deep integration of information technology and traditional agriculture, smart agriculture helps agricultural producers improve the yield per mu of land, stabilize product quality, reduce production costs, save natural resources, and reduce environmental pollution. In the past three years, with the improvement of sensor accuracy, big data, machine vision, machine learning and other fields have developed rapidly. In terms of fields, smart agriculture mainly covers precision agriculture, robots, drones, new farms, biological agriculture, and other aspects. From the perspective of solutions, it mainly includes remote intelligent agricultural monitoring, agricultural product safety traceability and anti-counterfeiting verification, standardized production of agricultural products, and brand marketing services of agricultural products. In foreign countries, Duffy and Regan (2017) indicated that based on the IoT technology, the autonomous sensor can be applied to the monitoring of phosphorus and nitrogen in water. Popovic et al. (2017) designed a platform for precise agricultural and ecological monitoring dedicated to the IoT, which can be applied to different servers and cloud technologies. Gupta \& Quamara (2018) indicated that the use of cloud computing to conduct statistics and analysis on a large number of data obtained by the IoT technology will become an important part of the future Internet.

In China, Liu et al. (2019) have shown that IoT technology has been applied in artificial light supplement, automatic mechanical arm sowing, harvesting, and other production processes. Li et al. (2017) have shown that IoT technology can be applied to real-time monitoring of temperature, humidity, light, soil PH value and carbon dioxide in agricultural environment. Zhu et al. (2017) showed that it is very important to apply wireless sensor networks in IoT to agricultural environment monitoring and data intelligent measurement. Mo's research shows that environment based on cloud computing can make the storage of big data in the IoT faster and safer by using enhanced ant colony algorithm (Mo, 2019). Based on the new generation of information technology, Liu et al. (2019) designed an integrated framework system platform integrating IoT, cloud computing, data mining, and other technologies. It shows that it can realize the basic functions of agricultural IoT monitoring through simulation. 
Combined with relevant research at home and abroad, it is found that with the continuous development of IoT technology, a large amount of data has been generated in different fields. Cloud computing can be used to effectively process and analyze these massive data, and the utilization rate of agricultural environmental information in foreign countries has been relatively wide, but at present, China's research on the combination of cloud computing, IoT and wireless sensor network technology and application in agricultural environmental information monitoring has decreased. Therefore, an agricultural environment IoT monitoring system was constructed based on cloud computing, aimed to lay a foundation for the study of real-time detection of the growing environment of crops by using IoT technology and cloud computing platform in the process of agricultural production.

\section{METHODOLOGY}

\subsection{Functional Structure of Agricultural Environment loT Monitoring System}

IoT is a system that senses connections between objects, including sensors, RFID, networking, transmission, processing, security, and data mining. The agricultural IOT information management system includes agricultural information perception, transmission, processing, and other modules. The sensing technology can collect and acquire the information required by the industrial system in any location by using sensors, GPS and RS, such as the light, temperature, humidity, water, carbon dioxide and ammonia in the environment. The transmission module can realize information transmission through wireless sensor networks or mobile communication technology based on ZigBee and other technologies. The processing of agricultural information can be realized through environmental prediction, early warning, optimal control, and intelligent decision-making. However, cloud computing is a method that can provide network access and configure network, server, storage, and other computing and computing sharing systems by providing availability and convenience quickly and according to different needs of users. Wolfert et al. (2017) believed that applying cloud computing to the management of IoT of agricultural information can maximize the utilization rate of resources, and has the advantages of providing more stable services, low-cost operation and accelerating the speed of agricultural development. According to the functional requirements, the monitoring system of the agricultural environment IOT can be divided into five major modules: intelligent gateway, device monitoring, data management, data analysis, and user center. There are corresponding submodules under each module. The specific functional structure is shown in Figure 1. The agricultural environment of Internet monitoring system mainly includes intelligent gateway, equipment monitoring, information management, data analysis and user center five levels of subsystem. The subsystems at these five levels contain 13 aspects including local monitoring, facility management, remote control, weather data, industry information, production management decisions, user management, and so on.

The architecture of the agricultural environment IoT monitoring system can be divided into four modules: perception layer, network layer, support layer, and application layer. Among them, the perception layer is mainly an intelligent gateway in the monitoring system, which can obtain, process and transfer sensors and other sensing data through connection. The internal sensor network of the gateway is implemented by ZigBee system. The network layer is mainly the gateway in the system and the network connection used for the transmission of data channels, usually the Internet and other networks. The support layer is the software and hardware environment that can be used for operation in the open system. This research adopts Amazon AWS cloud service platform to build the monitoring system. The application layer used Spring MVC framework in J2EE platform to realize the storage of perceptive data, device control, and data analysis.

\subsection{The Internet-Based Hierarchical Frame Design of the MONITORING System}

In order to realize the supervision and control of the agricultural production process, an information system of agricultural environment IOT monitoring was designed according to cloud computing. 


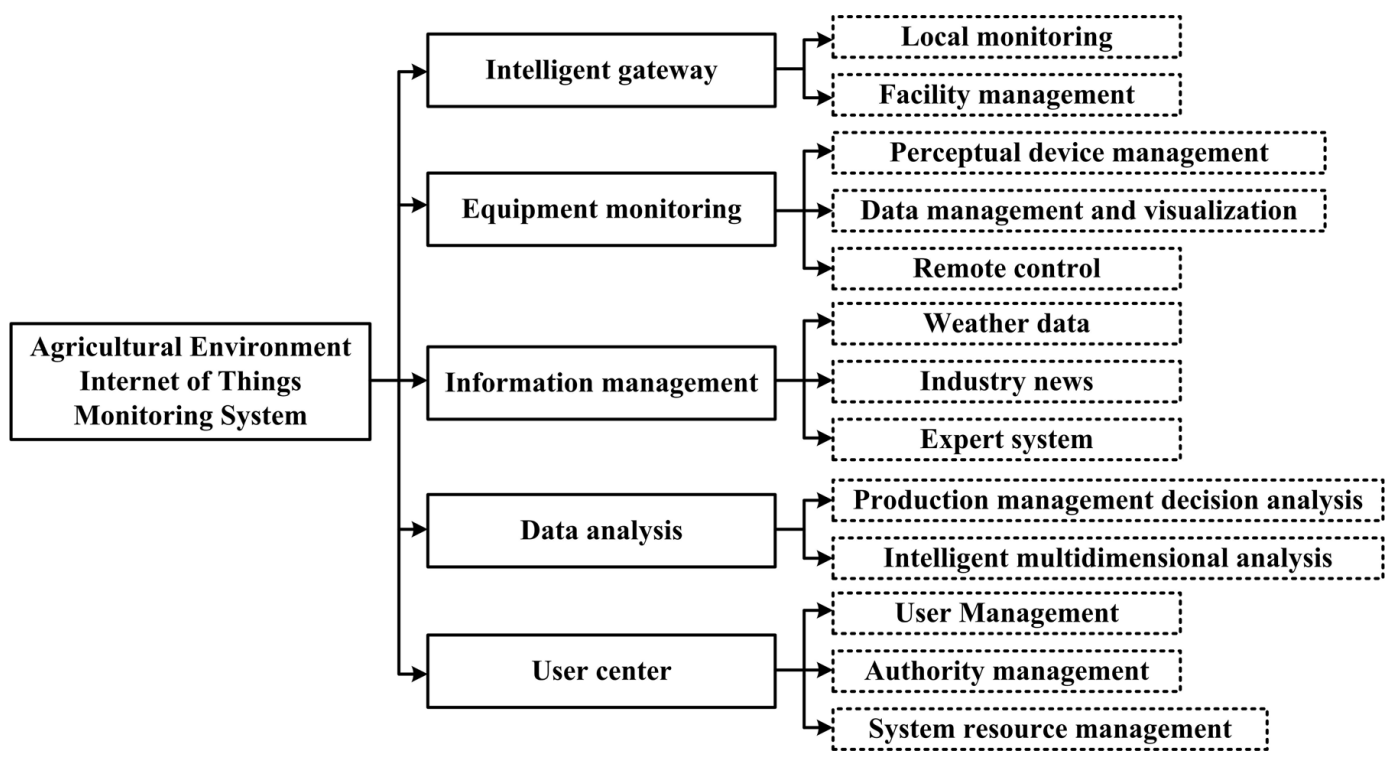

The system can collect and obtain the environmental information, the growth status of crops in the process of agricultural production through the sensor network, summarize and transmit it to the gateway of the IoT. Finally, decisions are made in view of the growth status of crops, so as to guide the agricultural production process. This research realizes the human-computer interaction, resource management, device control between users and the system on account of the user interface program and system support platform of Web browser. According to the main functions of the agricultural environment IoT monitoring system, it can be divided into four modules: equipment monitoring, data management, data analysis, and user center.

The monitoring of the equipment is mainly about data collection, processing, visualization and remote control of the equipment through the management perception equipment. Users can manage the perception of the equipment and obtain the data based on the RESful API resource structure. The data conforming to the trigger conditions are pushed through Websocket, and the system makes corresponding adjustments. The user can achieve remote control of the device by using the subscriber according to the MQTT protocol. The management of data is divided into weather data, industry news and expert system. The weather data can obtain professional weather prediction data and real-time monitoring data through API interface, and push it to big display in the user interface. Industry news can use Baidu and other news search engine equipment to obtain relevant agricultural information; Through the expert system, the user can directly consult the industry experts in the relevant fields on the technical issues related to agricultural technology. The data analysis module includes the production management decision analysis and intelligent multi-dimensional analysis, in which the production management decision can be obtained through mining, detection or network data to guide and supplement the existing management module. Intelligent multidimensional analysis can transform the data in the existing modules, and finally acquire knowledge related to production management to help make decisions. The user center includes user management, authority management, and resource management. Among them, user management is based on the SaaS service platform system to realize the authorization allocation of different users. Authority management using Restful API interface implementation; Resource management is based on the SaaS service platform system, which uses MQTT to push and count the resources stored in the system. The main system layered framework design is shown in Figure 2. 
Figure 2. Hierarchical framework design of agricultural environment loT monitoring system

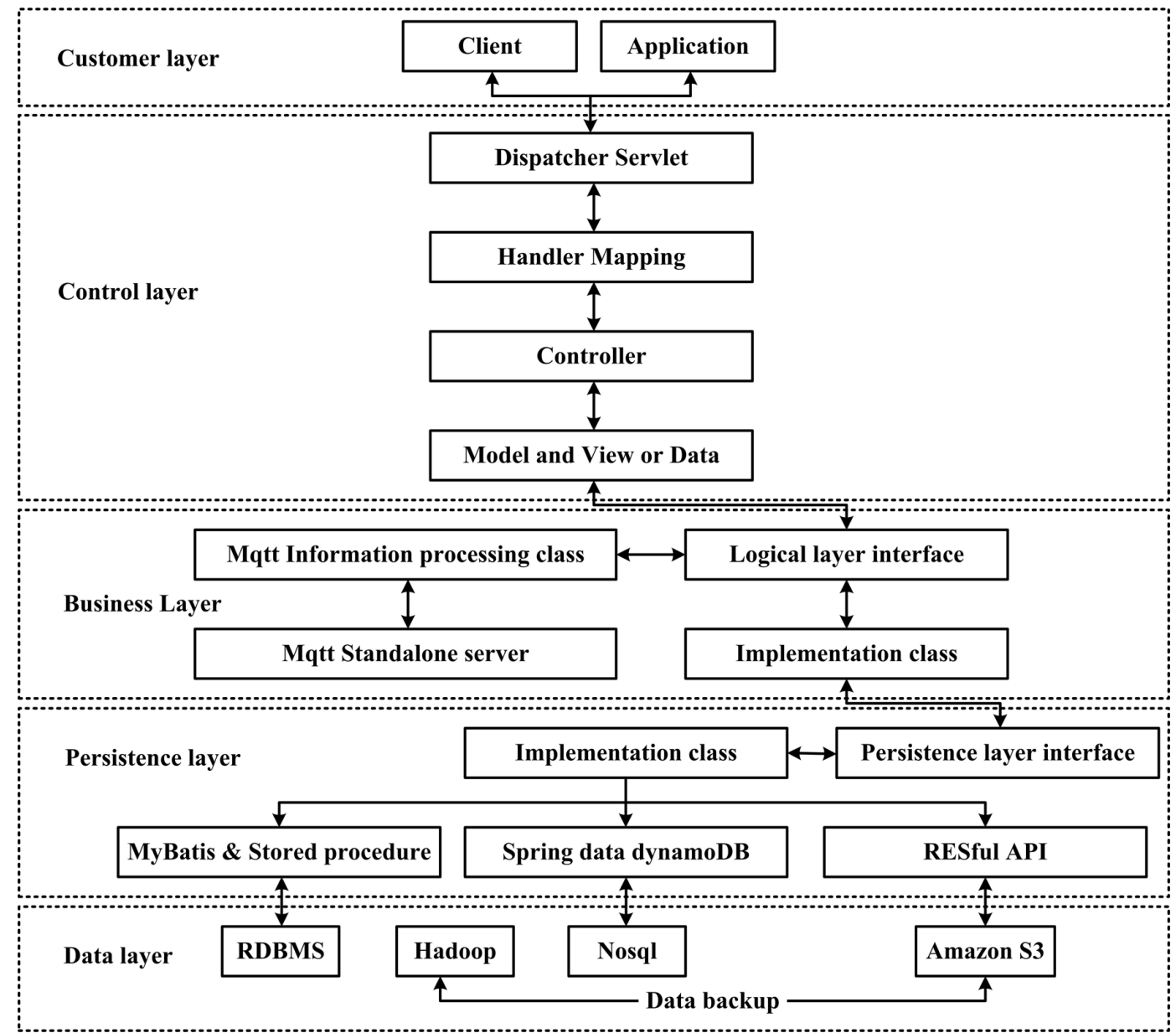

The traditional database storage system has gradually failed to meet the current requirements due to the large data collected. Therefore, the Amazon DynamoDB NoSQL database and RDS database were adopted to store data jointly. Spring Web MVC's basic framework based on Dispatcher Servlet is often applied to develop high-quality enterprise applications. MyBatis is a semi-automatic ORM framework, which can contact database and establish corresponding mapping. Spring data dynamo DB can make up for the disadvantages of MyBatis such as complex query and tedious writing SQL. Using the NoSQL cloud service database in Amazon DynamoDB, the database has the advantages of seamless expansion, high availability, strong security, and easy integration with Hadoop.AmazonS3 is a cloud-based storage service with a Web services interface and the ability to retrieve any amount of data in any location at a low cost. Hadoop framework can efficiently and conveniently realize the classification, transmission, and processing of massive data. It can interact with data in other storage services. According to Yu et al. (2018), MQTT is a communication protocol that can be used for lightweight or subscription information transmission, which can also provide reliable information transmission when the network communication signal is poor and unstable. 


\subsection{Intelligent Gateway System Design According to ZigBee}

The intelligent gateway module is an important part of the agricultural environment IoT system and an important hub for connecting the cognitive network and the communication network. According to the ZigBee sensor node, the agricultural environment monitoring system and the connection circuit between other modules and SIM900A module were designed. The hardware design of the intelligent gateway is to meet the monitoring requirements of factors such as temperature, humidity, illumination and soil moisture in the air, and to meet the detection of more different parameters. It is necessary to select a sensor that is lightweight, efficient, low-cost, short in delay, and highly expandable. Therefore, this study uses the GY-30 sensor to detect the illumination in the environment, the Risym sensor to detect the humidity in the soil, uses the DHT11 temperature and humidity sensor for the node construction, uses the SIM900A module based on STC12 microcontroller to collect and process the data collected by the sensor node, and finally uses the embedded ZigBee technology for data transmission. In order to improve the stability and detection efficiency of ZigBee remote monitoring system, ZigBee wireless sensor network with star topology structure is adopted in this study to collect diverse points and a large range of data and gather the data into the coordinator. The sink node transmits the data in the whole network to the single-chip microcomputer and then transmits the data remotely to the server through SIM900A module and stores it in the data, which is convenient for the data administrator and users to view. The software design of the intelligent gateway is based on the Linux kernel system. The RIP.GPIO and WiringPi are used to build the IO, I2C and SPI interfaces in the gateway. The UART port is built using the pyserial, the MQTT information is parsed and transmitted using paho-python, and the Web network service framework is built using WebIOPi. When using MQTT for remote control of the device, after receiving the command, the gateway will parse and verify the information, transmit the command to the ZigBee system through the serial port, and finally transmit the command to the terminal device for control by the ZigBee wireless transmission network.

\subsection{The overall Scheme Design Based on loT and Cloud Services}

In the cloud service platform, the base is one of the main data sources. The cloud service platform for big data can classify data by building multiple different types of databases. The framework of agricultural environmental information management system based on IoT and cloud services designed in this study is shown in Figure 3. Firstly, production information and visual information obtained by collection units such as wireless sensor networks as well as actuators including irrigation, temperature, humidity, light intensity and equipment control are required. Through the communication protocol converter of the cloud, the obtained data is transmitted to the cloud service management platform of agricultural environment big data based on cloud computing server, cloud platform, model library, database, decision library, and product library through wireless network. There is a two-way street between this cloud service platform and data acquisition. The cloud service platform can control the information collection equipment, intelligent control equipment, and visual monitoring equipment of the IoT. In addition, after information collection by various devices, the obtained data would be gathered through the bus to the communication protocol converter in the cloud for protocol conversion. The conversion Ethernet communication interface is uniformly connected to the Internet network, and the collected data is transferred to the cloud service platform through the network so that the user can directly realize the remote access of the cloud and the control of the device through the network.

Among them, mobile Internet dedicated line and telecommunication Internet dedicated line can have multiple Internet access through the link load balancer to route link traffic in Internet network and control broadband service level. 
Figure 3. Overall control scheme based on loT and remote cloud services

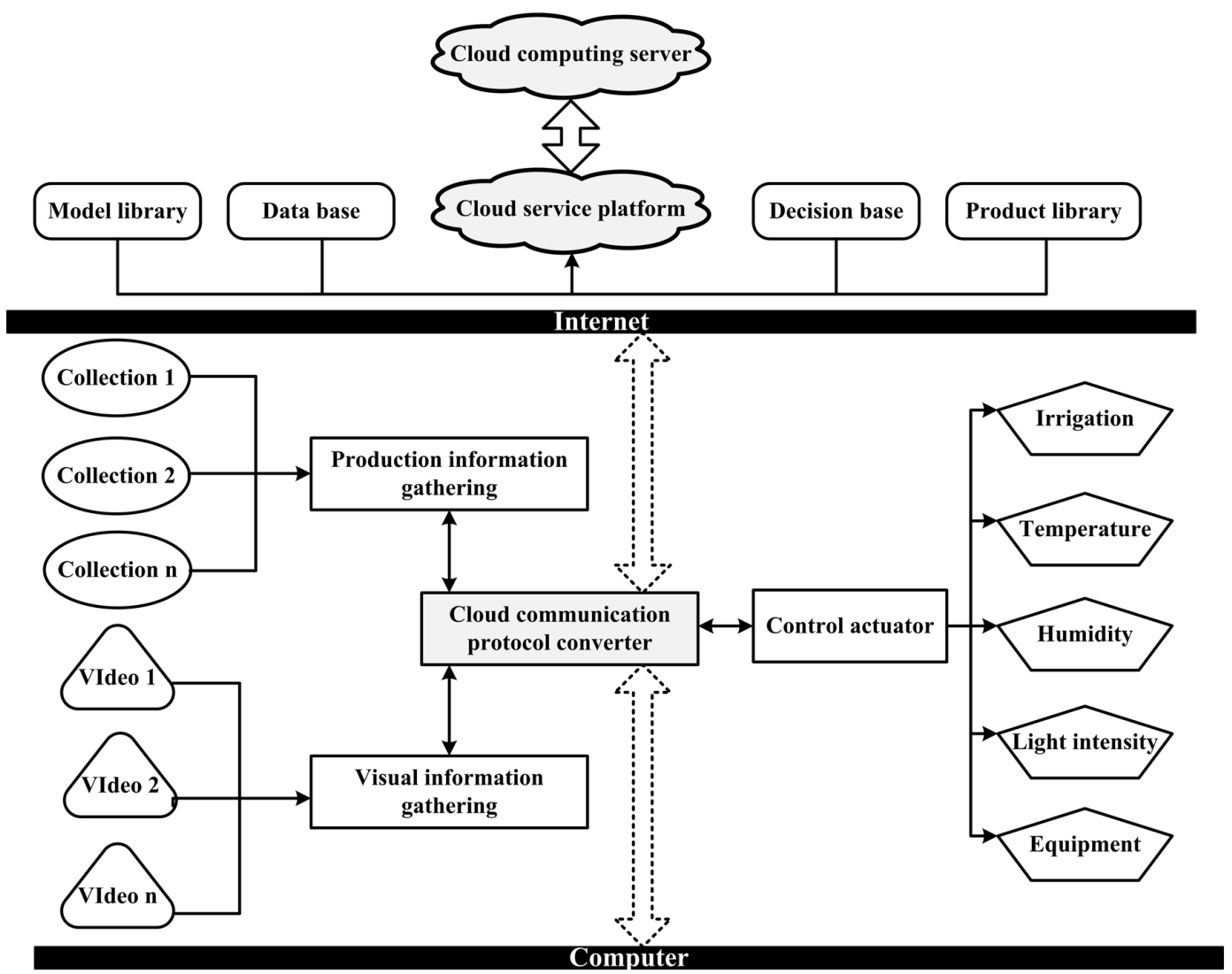

\subsection{Other Hardware and Software Design of the System}

Among them, the gy-30 module sensor of BH1750FVI chip is used for light detection, the CS215 signal sensor produced by Sensirion company is used for temperature and humidity detection, and the bm2000- $\mathrm{CO}_{2}$ sensor is used for $\mathrm{CO}_{2}$ detection. The specific parameters of all sensors are: temperature detection range: $0-50^{\circ} \mathrm{C}$; humidity detection range: $0-95 \%$; $\mathrm{CO}_{2}$ detection range: 0-50000ppm(adjustable); sampling time: $3 \mathrm{~s}$; response time: less than $60 \mathrm{~s}$; input power supply: 24 VDC; average power consumption: $100 \mathrm{~mA}$; accuracy: the measured value shall not exceed $5 \%$ of the actual value.

The architecture of cloud computing needs to be classified according to different types of services, which can be basically divided into infrastructure services, platform services and software services, and these applications need the support of a complete platform for operation. The hardware environment of the system also includes Http application server and Web server, etc., and the software environment includes specific operating system and database, etc., while the hardware and software can build the infrastructure of information technology. Therefore, the management platform of cloud agricultural environment data center is established, and the specific construction method is shown in Figure 4. It is mainly by installing the operating system; then the installation package of cloud agricultural environment data management platform is uploaded and stored in the root directory after installation; the Elaster file is extracted, then the management server software is installed, the install.sh script is executed, and M is selected; after installing MySQL database, the script is executed again and D is selected; the SELINUX variable is set to Permissive and the MySQL configuration 
Figure 4. The construction process of cloud agricultural environment data management platform
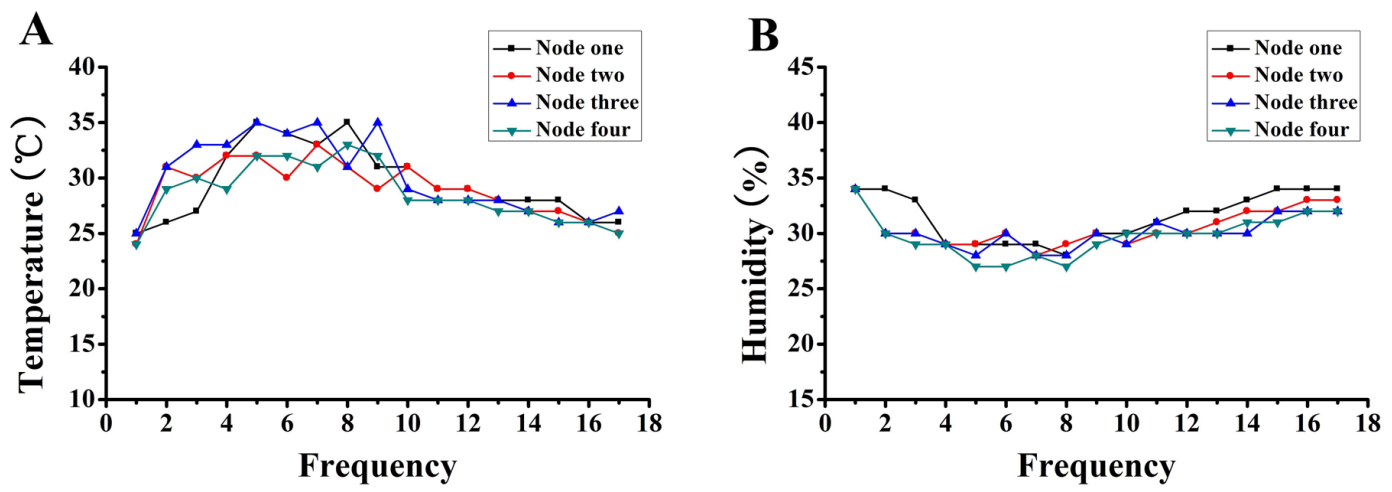

command is edited; after restarting MySQL service, user password is set; the cloud platform is started and accessed through the client.

\section{RESULTS}

In the Java environment, using the latest version of MyEclipse tools developed by J2EE, Maven tools for project management and code construction, using SVN for code version control. On the basis of the system is in the Spring MVC framework, between different groups as well as the framework through the way of integration, add it to Spring MVC framework, after loading the Spring configuration file, enter the code, the Web application context as the parent environment, using the definition parameters, data source, management and the framework of the Spring and the parameter is set with different database integration framework. MyBatis is defined as the data persistence layer framework, and the interface in the equipment management module is realized. Develop ORM framework in persistence layer in stored procedure to ensure automatic realization of Cursor data set and property mapping of specified entity class; Spring data dynamo DB combines with relevant functions to form an ORM framework to realize the storage of sensor nodes' perceived data. In the Java environment, provide a set of API functions, develop MQTT communication program calls, and use MQTT transmission information to achieve remote control of the agricultural environment IOT system; In order to ensure the safe operation of the system, the information transmission needs to go through the RESful interface for device management, data query and transmission, and user signature verification.

After debugging the system, the sensor nodes in the system were placed indoors for air temperature and humidity detection. The detection time ranged from 9:00 a.m. to P.M. 17:30 on July 10, and the detection interval was $0.5 \mathrm{~h}$. A total of 17 groups of data were obtained, and the test results were shown in Figure 3. Four sensor nodes were placed on the air temperature and humidity testing, from Figure $5 \mathrm{~A}$ and $5 \mathrm{~B}$, different node detection of temperature and humidity, there exist certain differences between but the difference is small, the changing trend of temperature and humidity and different time is consistent with the actual situation of temperature and humidity changes. The temperature showed a trend of first rising and then falling, while the humidity showed a trend of first falling and then rising. It indicates that real-time monitoring of environment-related parameters can be realized through the agricultural environment IoT monitoring system according to cloud computing.

Note: Figure A shows the detection results of the temperature of different sensor nodes at different time points; Figure B shows the humidity detection results of different sensor nodes at different time points.

The data obtained from the device end is transferred to the cloud through the network so that the agricultural environment data detection and intelligent control can be realized anywhere through the 
Figure 5. Temperature and humidity detection results at different time points and different sensor nodes
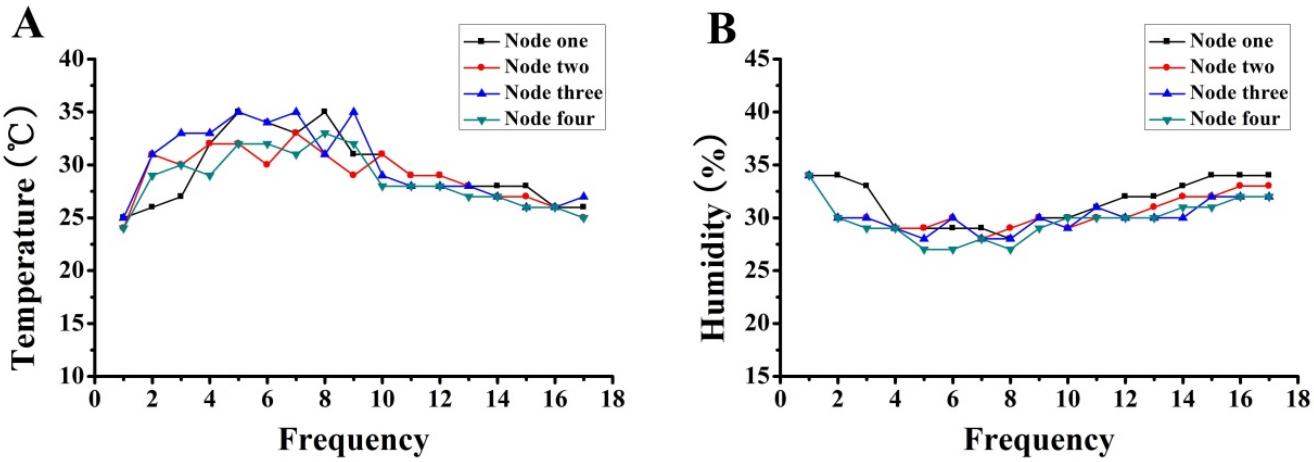

computer connected to the Internet. In addition, it can realize real-time monitoring of environmental information and visual information, and view the accumulation and change trend of environmental data in any time period. The control of temperature and humidity is one of the important indexes that affect the growth of crops. After the actual temperature and humidity in the environment are detected by sensors, the wireless transmission system can carry out independent temperature rise, temperature drop, spraying, irrigation, and other adjustment activities for environmental control. After the code program is written, the code is downloaded into the coordinator, routing node and terminal node respectively, and compiled. After there are no errors, each development board is powered on and the coordinator is connected to the computer using serial port line. At this time, the computer summary will display the data results collected by each node, as shown in Figure 6. Username and role, user account information, real-time data, historical data, statistics and analysis of data and office data

Figure 6 . The environmental data curve based on cloud access

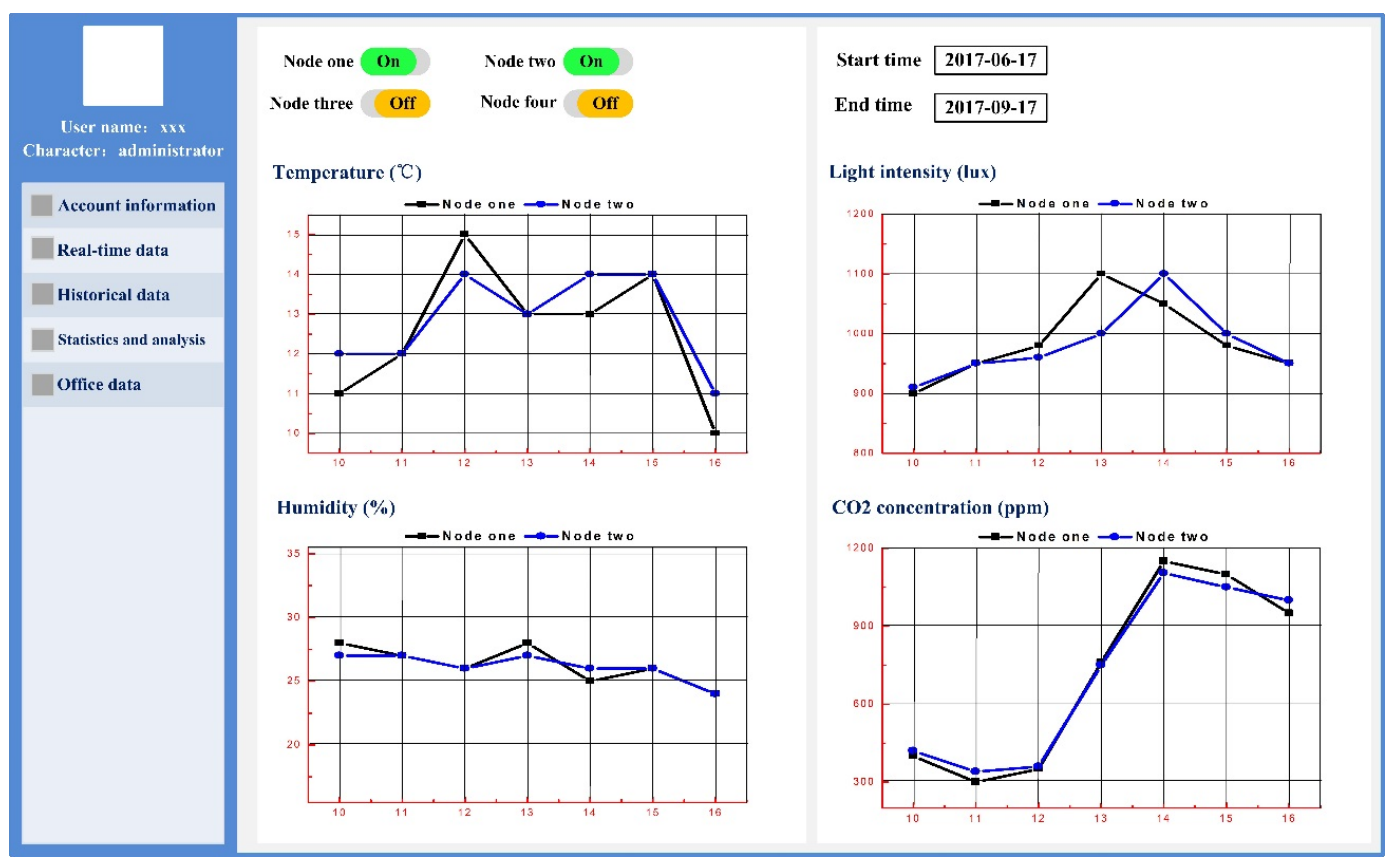


will appear in the web interface. It also displays information about air temperature, humidity, light intensity, and $\mathrm{CO}_{2}$ concentration.

\section{DISCUSSION}

As an important part of smart agriculture, agricultural intelligent monitoring is to set up "Internet of things" monitoring network by deploying cameras, sensors, collectors and other Internet of things devices in agricultural production sites. Using modern information technology and relying on computer or mobile phone terminal, real-time monitoring of climate environment, soil condition, crop growth, diseases and insect pests can be realized on agricultural production site. In addition, according to the preset rules, remote automatic control of various agricultural facilities and equipment is carried out to realize mass data collection and accurate control execution in agricultural production. Cloud computing is one of the distributed computing methods. It is usually required to divide the huge data into countless small programs through the network "Cloud" for calculation and processing. The system of multiple servers is then used to return the small programs that have been processed and analyzed to the user. According to Lewis (2017), its core concept is internet-centric, providing users with fast and secure cloud computing services and relevant data storage on the network so that every user can obtain resources through the Internet. Silva et al. (2017) pointed out that timely provision of relevant agricultural information is crucial for farmers to make correct and effective decisions, so they developed a mobile agricultural information system for farmers' daily decision-making. A large number of studies have shown that applying cloud computing to agricultural information management can effectively promote the development of the agricultural field. It is of great significance to use the Internet of things to build a real-time monitoring system of agricultural environment information for us to know the agricultural environment information timely and in real time, and then put forward accurate and correct decisions. Therefore, a real-time monitoring system of agricultural environment information is designed based on the Internet of things. Chen et al. (2018) found that the application of IOT technology to the fine control of fertilizers in agriculture based on cloud platform could realize the processing of massive data and provide effective solutions for the selection, production and cultivation management of crop varieties. Cheng et al. (2012) proposed a framework based on cloud computing and ZigBee remote monitoring and control, while an agricultural environment information management system framework was designed based on IoT and cloud services. Among them, ZigBee wireless sensor is used to realize real-time monitoring of environmental information. After the test, it was found that it could effectively monitor the changes of temperature and humidity in the air, and there was little difference between the nodes, indicating that better detection results could be obtained by real-time monitoring of ambient temperature and humidity with the sensor node network (Syafarinda et al., 2018). The change of temperature and humidity in the air detected by the node shows the opposite trend. This is because the constant increase of solar radiation intensity will gradually increase the temperature in the air, but also increase the evaporation degree of water in the air. If the humidity in the air is lower than the optimum growth humidity of crops, spray or irrigation is needed. Sheng \& Cai (2016) combined ZigBee technology, wireless sensor network, and cloud computing technology to design a framework for remote monitoring of temperature. The results showed that rapid query and control of environmental temperature could be realized through the Web or personal mobile terminals. Yang et al. (2018) proposed an intelligent indoor environment monitoring system combined with ZigBee wireless sensor network technology. Finally, the result information is provided through the web-based monitoring platform, so that users can use the Internet to monitor the environment and enable them to use these wise decisions to manage and improve the environment. In this study, ZigBee and wireless sensor networks were combined to build an agricultural environment information management system. Finally, it was found that it could monitor the temperature, humidity, light intensity and $\mathrm{CO}_{2}$ concentration in the agricultural 
environment in real-time, which laid a foundation for the subsequent mining of data related to the agricultural environment.

\section{CONCLUSION}

The cloud IOT platform is mainly designed to solve the information management of the agricultural environment, and users can enter the base end through the large platform. Different bases can carry out different accounts and login conditions, so as to realize the flexible management of the agricultural IOT cloud platform and ensure the security of the platform. The general framework structure of the modern agricultural IoT monitoring system is proposed and designed according to cloud computing through the modern agricultural IOT monitoring system, intelligent gateway of IoT, and MQTT information service. The Spring MVC basic framework based on J2EE platform is designed, and the modules such as MyBatis in the cloud service platform are integrated into the framework. In view of ZigBee communication technology, the information transmission inside the intelligent gateway of agricultural environment IoT is realized. The information transmission server according to MQTT information protocol is set up to realize the connection of agricultural environment IOT and intelligent gateway. Finally, through the verification test, it is found that the system can realize the real-time monitoring, analysis and storage functions of environmental data, and the monitoring results are relatively accurate. Besides, the corresponding instructions can be sent. Subsequently, in order to promote the management and intelligent control of agricultural environmental information, the construction of intelligent control system in the environment can be carried out, such as carbon dioxide supply system and automatic nutrient solution supply system. To sum up, this system lays a foundation for the effective management of agricultural production process. 


\section{REFERENCES}

Li, M., Chen, S., Liu, F., Zhao, L., Xue, Q., Wang, H., ... Bienvenido, J. F. (2017). A risk management system for meteorological disasters of solar greenhouse vegetables. Precision Agriculture, (3): 1-14.

Chen, J. B., Cao, X. L., Fu, H. C., \& Lam, A. (2018). Agricultural product monitoring system supported by cloud computing. Cluster Computing, (3): 1-10.

Cheng, J. Y., Hung, M. H., Lin, S. S., \& Cheng, F. T. (2012). New remote monitoring and control system architectures based on cloud computing. Advanced Materials Research, 579, 312-329. doi:10.4028/www. scientific.net/AMR.579.312

De Silva, L. N. C., Goonetillake, J. S., Wikramanayake, G. N., \& Ginige, A. (2017, May). Harnessing mobile pervasive computing to enhance livelihood processes: Farmer response to a mobile agriculture information system. In Proceedings of the International Conference on Green, Pervasive, and Cloud Computing (pp. 641655). Springer.

Duffy, G., \& Regan, F. (2017). Recent developments in sensing methods for eutrophying nutrients with a focus on automation for environmental applications. Analyst (London), 142(23), 4335-4372. doi:10.1039/C7AN00840F PMID:28765839

Gupta, B. B., \& Quamara, M. (2018). An identity-based access control and mutual authentication framework for distributed cloud computing services in iot environment using smart cards. Procedia Computer Science, 132, 189-197. doi:10.1016/j.procs.2018.05.185

Lai, W. Y. (2017). Pesticide use and health outcomes: Evidence from agricultural water pollution in China. Journal of Environmental Economics and Management, 86, 93-120. doi:10.1016/j.jeem.2017.05.006

Lewis, G. A. (2017). Cloud computing. Computer, 50(5), 8-9. doi:10.1109/MC.2017.141

Liu, D., Gong, Y., Wang, G., Chen, X., Zhang, X., \& Wu, G. (2018). Research Advances in Mechanization of Pesticide Spraying Technology for Facility Agriculture. Asian Agricultural Research, 10(7), 81-86.

Liu, S., Guo, L., Webb, H., Yao, X., \& Chang, X. (2019). IOT monitoring system of modern eco-agriculture based on cloud computing. IEEE Access : Practical Innovations, Open Solutions, 7(99), 1-1.

Mo, Y. (2019). A data security storage method for iot under hadoop cloud computing platform. International Journal of Wireless Information Networks, 26(2), 152-157. doi:10.1007/s10776-019-00434-x

Popovic, T., Latinovic, N., Pesic, A., Zecevic, Z., \& Djukanovic, S. (2017). Architecting an iot-enabled platform for precision agriculture and ecological monitoring: A case study. Computers and Electronics in Agriculture, 140(140C), 255-265. doi:10.1016/j.compag.2017.06.008

Sheng, X. N., \& Cai, S. M. (2016). Zigbee and cloud computing-based temperature monitoring and control system architecture design. Forestry Machinery \& Woodworking Equipment.

Syafarinda, Y., Akhadin, F., Fitri, Z. E., \& Widiawan, B. (2018). The precision agriculture based on wireless sensor network with MQTT protocol. IOP Conference Series Earth and Environmental Science, 207, 012059. doi:10.1088/1755-1315/207/1/012059

Wang, L., Zhou, H., Guo, S., An, Y., Shu, S., Lu, N., \& Sun, J. (2018). Exogenous spermidine maintains the chloroplast structure of cucumber seedlings and inhibits the degradation of photosynthetic protein complexes under high-temperature stress. Acta Physiologiae Plantarum, 40(3), 47. doi:10.1007/s11738-018-2624-9

Wang, Y., He, J., Wang, P., Dai, J., Yang, B., Tan, H., \& Tao, G. (2017). Risk analysis of big data based on cloud computing for the inspection and testing of toxic and hazardous substances in meat products. Asian Agricultural Research, (9): 95-100.

Wolfert, S., Ge, L., Verdouw, C., \& Bogaardt, M.-J. (2017). Big data in smart farming - a review. Agricultural Systems, 153, 69-80. doi:10.1016/j.agsy.2017.01.023

Yang, C. T., Chen, S. T., Den, W., Wang, Y. T., \& Kristiani, E. (2018). Implementation of an intelligent indoor environmental monitoring and management system in cloud. Future Generation Computer Systems, 96, 731-749. doi:10.1016/j.future.2018.02.041 
Yu, J., Lim, J., \& Lee, K. S. (2018). Investigation of drought-vulnerable regions in North Korea using remote sensing and cloud computing climate data. Environmental Monitoring and Assessment, 190(3), 126. doi:10.1007/ s10661-018-6466-0 PMID:29423574

Zhang, R., Hao, F., \& Sun, X. (2017). The design of agricultural machinery service management system based on IOT. Procedia Computer Science, 107, 53-57. doi:10.1016/j.procs.2017.03.055

Zhu, J., Song, Y., Jiang, D., \& Song, H. (2017). Multi-armed bandit channel access scheme with cognitive radio technology in wireless sensor networks for the IOT. IEEE Access: Practical Innovations, Open Solutions, 4, 4609-4617. doi:10.1109/ACCESS.2016.2600633 